\title{
The Mycobacterium avium subsp. paratuberculosis fibronectin attachment protein, a toll-like receptor 4 agonist, enhances dendritic cell-based cancer vaccine potency
}

\author{
Kyung Tae Noh ${ }^{1 *}$, Sung Jae Shin ${ }^{3,4 *}$, \\ Kwang Hee Son ${ }^{1}$, In Duk Jung ${ }^{1}$, Hyun Kyu Kang ${ }^{1}$, \\ Su Jung Lee ${ }^{1}$, Eun Kyung Lee ${ }^{5}$, Yong Kyoo Shin ${ }^{6}$, \\ Ji Chang You ${ }^{7}$ and Yeong-Min Park ${ }^{1,2,8}$ \\ ${ }^{1}$ Department of Microbiology and Immunology \\ School of Medicine \\ Pusan National University \\ Yangsan 626-870, Korea \\ ${ }^{2}$ Research Institute of Convergence of \\ Biomedical Science and Technology \\ Pusan National University \\ Yangsan 626-770, Korea \\ ${ }^{3}$ Department of Microbiology \\ College of Medicine \\ Chungnam National University \\ Daejeon 301-747, Korea \\ ${ }^{4}$ Department of Microbiology \\ Institute of Immunology and Immunological Diseases \\ Yonsei University College of Medicine \\ Seoul 120-752, Korea \\ ${ }^{5}$ Yongsan Hospital College of Medicine \\ Chung-Ang University \\ Seoul 140-757, Korea \\ ${ }^{6}$ Department of Pharmacology \\ College of Medicine \\ Chung-Ang University \\ Seoul 156-756, Korea \\ ${ }^{7}$ National Research Laboratory of Molecular Virology \\ Department of Pathology \\ School of Medicine \\ The Catholic University of Korea \\ Seoul 137-701, Korea \\ ${ }^{8}$ Corresponding author: Tel, 82-51-510-8097; \\ Fax, 82-55-382-8090; E-mail, immunpym @ pusan.ac.kr \\ *These authors contributed equally to this work. \\ http://dx.doi.org/10.3858/emm.2012.44.5.038
}

Accepted 8 February 2012

Available Online 9 February 2012

Abbreviations: BMDC, bone marrow-derived dendritic cell; FAP, fibronectin attachment protein; GSK-3, glycogen synthase kinase-3; MLR, mixed lymphocyte reaction; TLR4, toll-like receptor 4

\begin{abstract}
In this study, we showed the direct interaction between Mycobacterium avium subsp. paratuberculosis fibronectin attachment protein (FAP) and toll-like receptor4 (TLR4) via co-localization and binding by using confocal microscopy and co-immunoprecipitation assays. FAP triggered the expression of pro- and antiinflammatory cytokines in a TLR4-dependent manner. In addition, FAP-induced cytokine expression in bone marrow-derived dendritic cells (BMDCs) was modulated in part by glycogen synthase kinase-3 (GSK-3). FAP-induced expression of CD80, CD86, major histocompatibility complex (MHC) class I, and MHC class II in $T L R 4^{++}$BMDCs was not observed in TLR4 ${ }^{-/}$BMDCs. Furthermore, FAP induced DC-mediated $\mathrm{CD8}^{+} \mathrm{T}$ cell proliferation and cytotoxic T lymphocyte (CTL) activity, and suppressed tumor growth with DC-based tumor vaccination in EG7 thymoma murine model. Taken together, these results indicate that the TLR4 agonist, FAP, a potential immunoadjuvant for DC-based cancer vaccination, improves the DC-based immune response via the TLR4 signaling pathway.
\end{abstract}

Keywords: dendritic cells; FAP-A protein, Mycobacterium avium; glycogen synthase kinase-3; toll-like receptor 4

\section{Introduction}

Dendritic cells (DCs), which are representative antigen-presenting cells (APCs), play a pivotal role in the modulation of the immune response to various antigens via toll-like receptors (TLRs) (Watts et al., 2010). Immature DCs capture and process antigens in the peripheral tissues (Banchereau et al., 2000). After antigen uptake, mature DCs migrate to draining lymph nodes, wherein they activate naïve T-cells by upregulating surface molecules, including CD80, CD86, major histocompatibility complex (MHC) class $\mathrm{I}$, and MHC class II (Alvarez et al., 2008). In addition, 
mature DCs can secrete various immunoregulatory cytokines such as interleukin (IL)-12, IL-6, and IL-10, which are regulators of inflammatory responses, and activate innate immune cells (Jensen and Gad, 2010). Thus, it is well accepted that DC serves as a crucial linker between innate and adaptive immune response (Liu, 2001). The activation of nuclear factor kappa B (NF-kB) and mitogen-activated protein kinases (MAPKs), including extracellular signal-regulated kinase (ERK), c-jun N-terminal kinase (JNK), and p38, via TLRs regulates the maturation and differentiation of DCs (Rescigno et al., 1998).

The recognition of microbial products such as lipopolysaccharide (LPS) is initiated by TLRs, which recruit various signaling molecules, including the adaptor molecules myeloid differentiation protein 88 (MyD88), tumor necrosis factor receptor-associated factor 6 (TRAF6) and MyD88 adapter-like (MAL), resulting in the activation of downstream signaling pathways such as MAPKs and NF-kB (Akira et al., 2001). Because TLR activation usually polarizes Th1 responses, TLR agonists are potent candidates for immune adjuvants in tumor therapy (Matijevic and Pavelic, 2010). Among these mentioned TLR agonists, TLR4 agonists are more suitable for immunostimulatory adjuvants due to the DC-based immune boosting potency via TLR4 signaling cascade (Napolitani et al., 2005).

Glycogen synthase kinase-3 (GSK-3) was first identified as a key regulator of the glycogen metabolism; it regulates various cellular processes such as apoptosis, differentiation, growth, cell motility, and embryonic development by modulating various substrates (Cohen and Frame, 2001; Woodgett, 2001; Jope and Johnson, 2004). Recent studies have shown the role of GSK-3 as a regulator of immune responses, including activation and differentiation of DCs and endotoxemia (Rodionova et al., 2007; Noh et al., 2011).

Fibronectin (FN) attachment proteins (FAPs) belong to a family of $\mathrm{FN}$-binding glycoproteins that are expressed by several species of mycobacteria (Ratliff et al., 1993; Schorey et al., 1995, 1996; Secott et al., 2001). FAP is crucial for attachment and internalization of Mycobacterium bovis BCG and $M$. avium subsp. avium to tissue of host (Middleton et al., 2000; Zhao et al., 2000). These processes were proved to be a FAP-dependent process (Kuroda et al., 1993; Schorey et al., 1995). M. avium subsp. paratuberculosis also expresses a FAP which mediates soluble FN binding (Secott et al., 2001). In a previous report, we had shown that FAP expressed by $M$. avium enhances the immune response via regulation of DCs (Lee et al.,
A

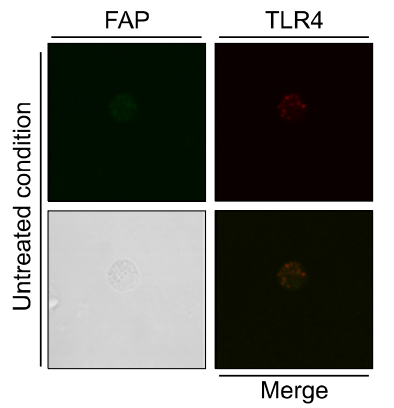

B

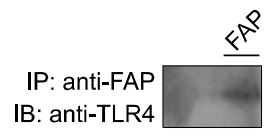

IB: anti-TLR4

IB: anti-FAP

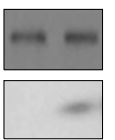

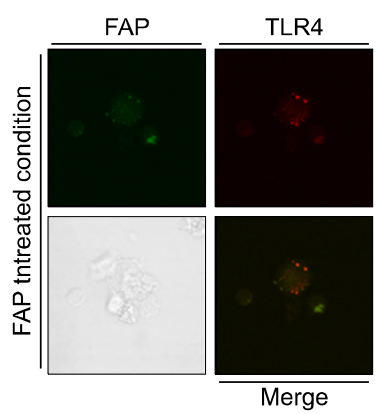

Figure 1. FAP interacts with TLR4. (A) Co-localization of FAP and TLR4 on DCs. DCs were treated with FAP ( $500 \mathrm{ng} / \mathrm{ml})$ for $30 \mathrm{~min}$, fixed, and stained with rabbit anti-FAP and mouse PE-conjugated anti-TLR4 antibodies overnight at $4^{\circ} \mathrm{C}$, and then stained with Alexa568-conjugated anti-rabbit antibody for $1 \mathrm{~h}$ at room temperature. Cell morphology and fluorescence intensity were analyzed using the Zeiss LSM510 Meta confocal laser scanning microscope. (B) Co-immunoprecipitation of FAP and TLR4. DCs were treated with FAP $(500 \mathrm{ng} / \mathrm{ml})$ for $30 \mathrm{~min}$. Cells were harvested, cell lysates were immunoprecipitated with $\lg G$ and anti-FAP, and proteins were visualized by immunoblotting with an anti-TLR4 antibody.

2009). However, the detailed mechanism of the FAP-regulated immune response is less defined.

In this study, we have shown the direct interaction between FAP and TLR4 in BMDCs. The expression of surface markers, including CD80, CD86, MHC class I, and MHC class II, and pro- and anti-inflammatory cytokines by FAP is TLR4dependently modulated. In addition, we identified that $\mathrm{DC}$-mediated $\mathrm{CD} 8^{+} \mathrm{T}$ cell proliferation and cytotoxic T lymphocyte activity by FAP is via GSK-3.

\section{Results}

\section{FAP interacts with TLR4 in BMDCs}

Previously, it was shown that $M$. avium regulates the immune response through modulation of DC maturation (Lei and Hostetter, 2007). Furthermore, we determined that the $M$. avium protein FAP is quite effective in inducing DC maturation and Th1 polarization (Lee et al., 2009). However, the precise mechanism by which this antigen mediates immune stimulation has not been defined. A Th1 
A
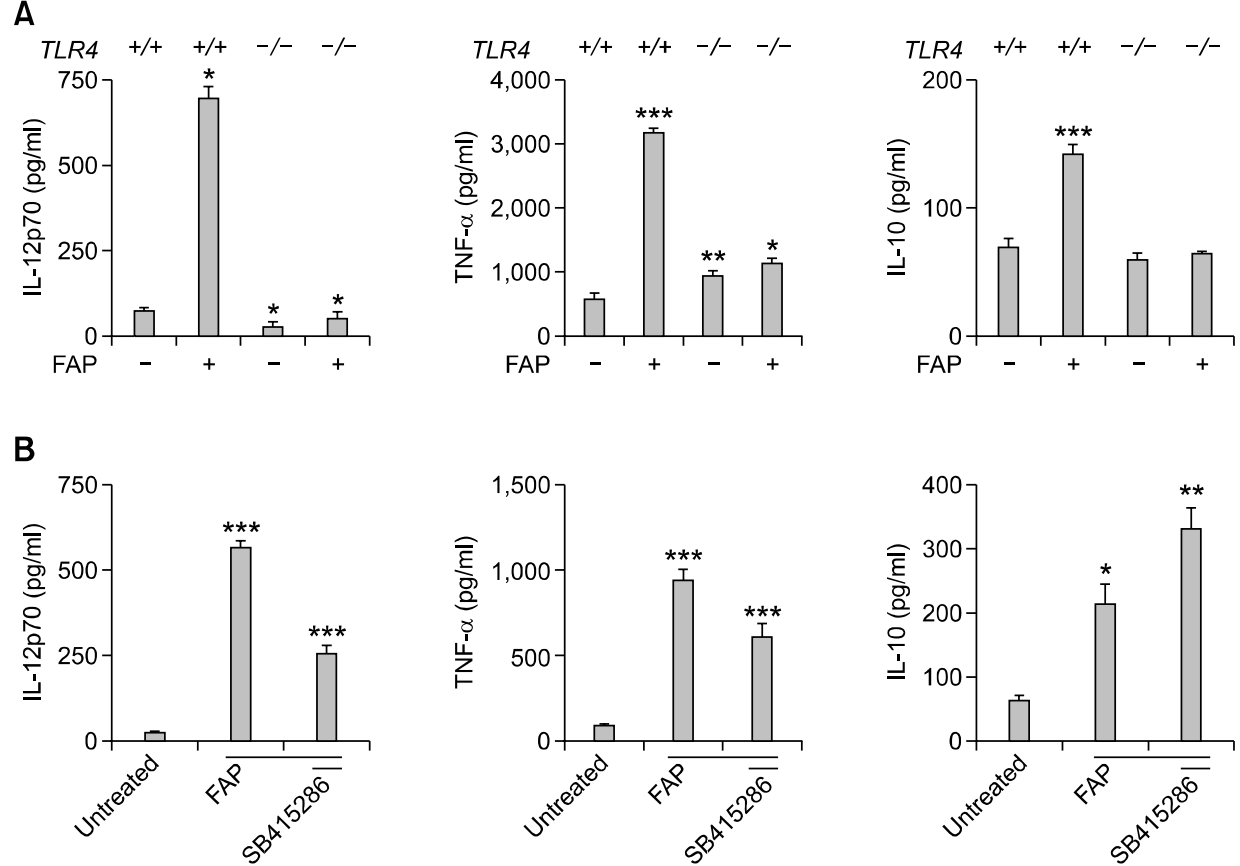

Figure 2. The expression of the pro-inflammatory cytokines (IL-12 and TNF- $\alpha$ ) and the anti-inflammatory cytokine IL-10 was enhanced by FAP treatment

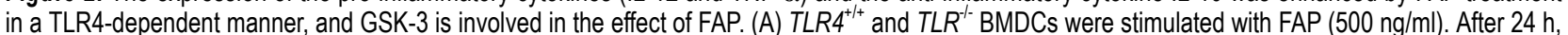
the concentrations of IL-12, TNF- $\alpha$, and IL-10 were measured in the culture supernatants by ELISA. The mean (SEM) values shown represent 3 independent experiments. ${ }^{*} P<0.05,{ }^{* *} P<0.01,{ }^{* * *} P<0.001$ compared with untreated TLR4 ${ }^{+/+}$BMDCs. (B) BMDCs were pretreated with or without the indicated GSK-3 inhibitor (SB415286) concentration for $30 \mathrm{~min}$ and then harvested after $24 \mathrm{~h}$ of incubation with FAP (500 $\mathrm{ng} / \mathrm{ml})$. The cytokine concentrations in culture supernatants were measured by ELISA. The mean \pm SEM values shown represent 3 independent experiments. ${ }^{*} P<0.05,{ }^{* *} P<$ $0.01,{ }^{* * *} P<0.001$ compared with untreated BMDCs.

response is usually triggered by TLR activation in dendritic cells. Using confocal microscopy and co-immunoprecipitation assays, we found that FAP co-localizes and binds with TLR4 in BMDCs (Figure 1). These data demonstrates that FAP interacts with TLR4. Next, we investigated whether FAP plays a role as one of the TLR4 ligands by using TLR4 $4^{-/-}$BMDCs. FAP activated MAPKs, including ERK, JNK, and $p 38$, and $N F-\kappa B$ in the presence of TLR4, but this phenomenon was not observed in cells in which TLR4 was knocked out (Supplemental Data Figure S1).

\section{The expression of the pro-inflammatory cytokines (IL-12 and TNF- $\alpha$ ) and the anti-inflammatory cytokine IL-10 was enhanced by FAP treatment in a TLR4- dependent manner, and GSK-3 is involved in the effect of FAP}

Next, we investigated whether stimulation of DCs with FAP affects the production of pro- and anti-inflammatory cytokine that modulate the polarization of Th1 and Th2 cells via TLR4. We analyzed the production of the pro-inflammatory cytokines IL-12 and TNF- $\alpha$ and that of the antiinflammatory cytokine IL-10 in FAP-treated $T L R 4^{+/+}$ and TLR4 $4^{-/}$BMDCs using the ELISA method. FAP treatment elevated the production of the pro-inflammatory cytokines IL-12 and TNF- $\alpha$ as well as that of the anti-inflammatory cytokine IL-10, but the increase in the production of those cytokines by FAP was impaired in the absence of TLR4 (Figure 2A). Thus, the FAP-mediated cytokine production, which is crucial for the modulation of DC functions, was also regulated in a TLR4dependent manner.

In a recent publication, M. Martin et al. provided an evidence that GSK-3 modulates various cytokines, including IL-12 and TNF- $\alpha$, via TLR4 (Martin et al., 2005). Thus, we examined whether GSK-3 is involved in the modulation of the abovementioned cytokines in the presence of FAP. Inhibition of GSK-3 resulted in the reduction of the IL-12 and TNF- $\alpha$ production compared with the FAP-treated control, whereas the IL-10 production was dramatically enhanced (Figure 2B). This result demonstrates that GSK-3 is one of signaling pathway involved in the cytokine modulation by FAP. 
A
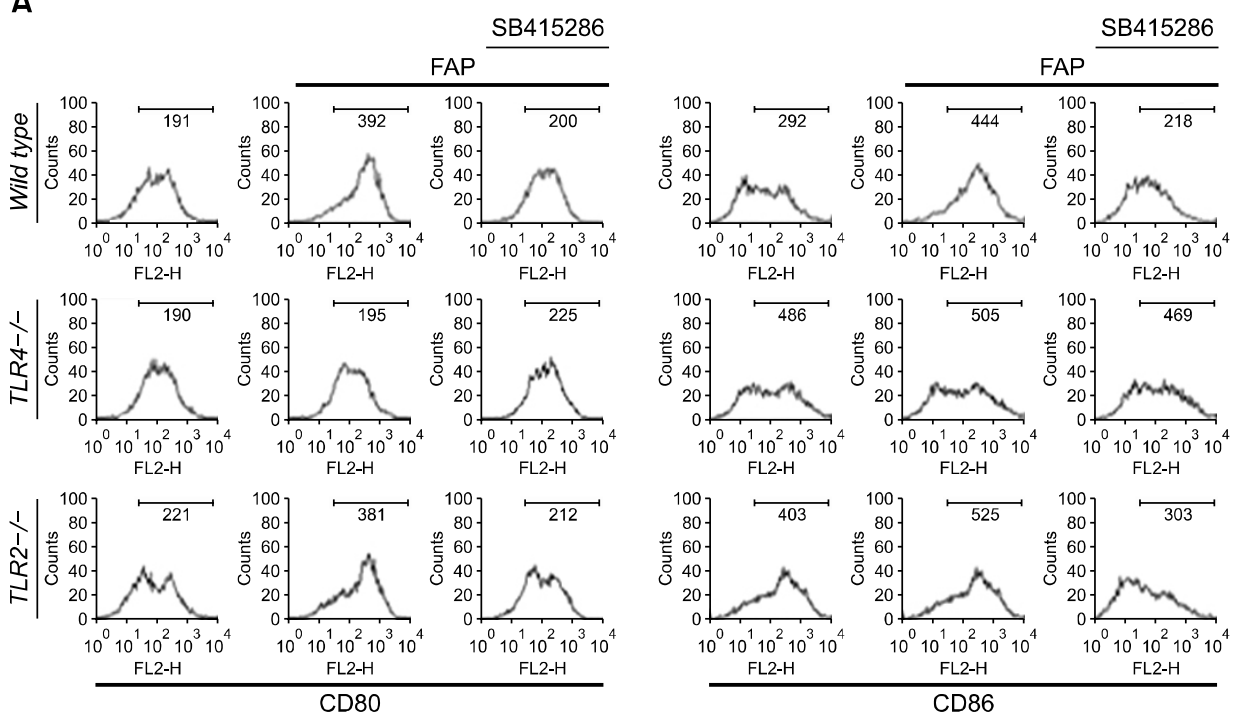

Figure 3. FAP induces the expression of $\mathrm{MHC}$ class I and II and co-stimulatory molecules (CD80 and CD 86) in wild type and $T L R 2^{-1 .}$ BMDCs, but the expression of those molecules was impaired in TLR4 BMDCs. On day 6 of culturing, BMDCs were pretreated with or without the indicated GSK-3 inhibitor (SB415286) concentration for 30 min and then harvested after $24 \mathrm{~h}$ of incubation with FAP $(500 \mathrm{ng} / \mathrm{ml})$. BMDCs were first gated for $\mathrm{CD} 11 \mathrm{c}^{+}$ cells and analyzed by two-color flow cytometry. (A) FAP induces the expression of co-stimulatory molecules (CD80 and CD86) in wild type and TLR2 ${ }^{--}$BMDCs, but the expression of these molecules was impaired in TLR4 ${ }^{-1-}$ BMDCs. The mean fluorescence intensity (MFI) of double-positive cells is shown for each panel. The mean \pm SEM values shown represent 3 independent experiments. ${ }^{* *} P<0.01$ compared with untreated BMDCs. (B) FAP induces expression of MHC class I and II in wild type and $T L R 2^{-1}$ BMDCs, but the expression of these molecules was impaired in $T L R 4^{-1}$ BMDCs. The mean fluorescence intensity (MFI) of double-positive cells is shown for each panel. The mean \pm SEM values shown represent 3 independent experiments. ${ }^{*} P<0.05$ compared with untreated BMDCs.

FAP induces the expression of co-stimulatory (CD80 and $C D$ 86) and MHC class I and II molecules in TLR4 ${ }^{++}$ BMDCs, but the expression of those molecules was impaired in $\mathrm{TLR}^{-\mathrm{r}} \mathrm{BMDCs}$

The activation of TLR4, one of the crucial proteins in innate and adaptive immune responses, by various stimuli leads to the maturation of DCs. Thus, we examined the effect of FAP on DC maturation by investigating the expression of surface markers using flow cytometry. We found increased expression of surface markers, including CD80, CD86, MHC class I, and MHC class II, in

FAP-treated $T L R 4^{+/+}$BMDCs, but this phenomenon was not observed in FAP-treated TLR4 $4^{-1-}$ BMDCs (Figure 3). However, the expression of the abovementioned surface markers was still activated by FAP in $T L R 2^{-1-}$ BMDCs (Figure 3). In addition, FAP-mediated DC maturation was impaired in GSK-3 inhibitor-treated condition. From this result, we concluded that DC maturation mediated by FAP occurs via the TLR4 rather than the TLR2 signaling pathway, and FAP-mediated DC maturation is partly dependent on GSK-3. 
B
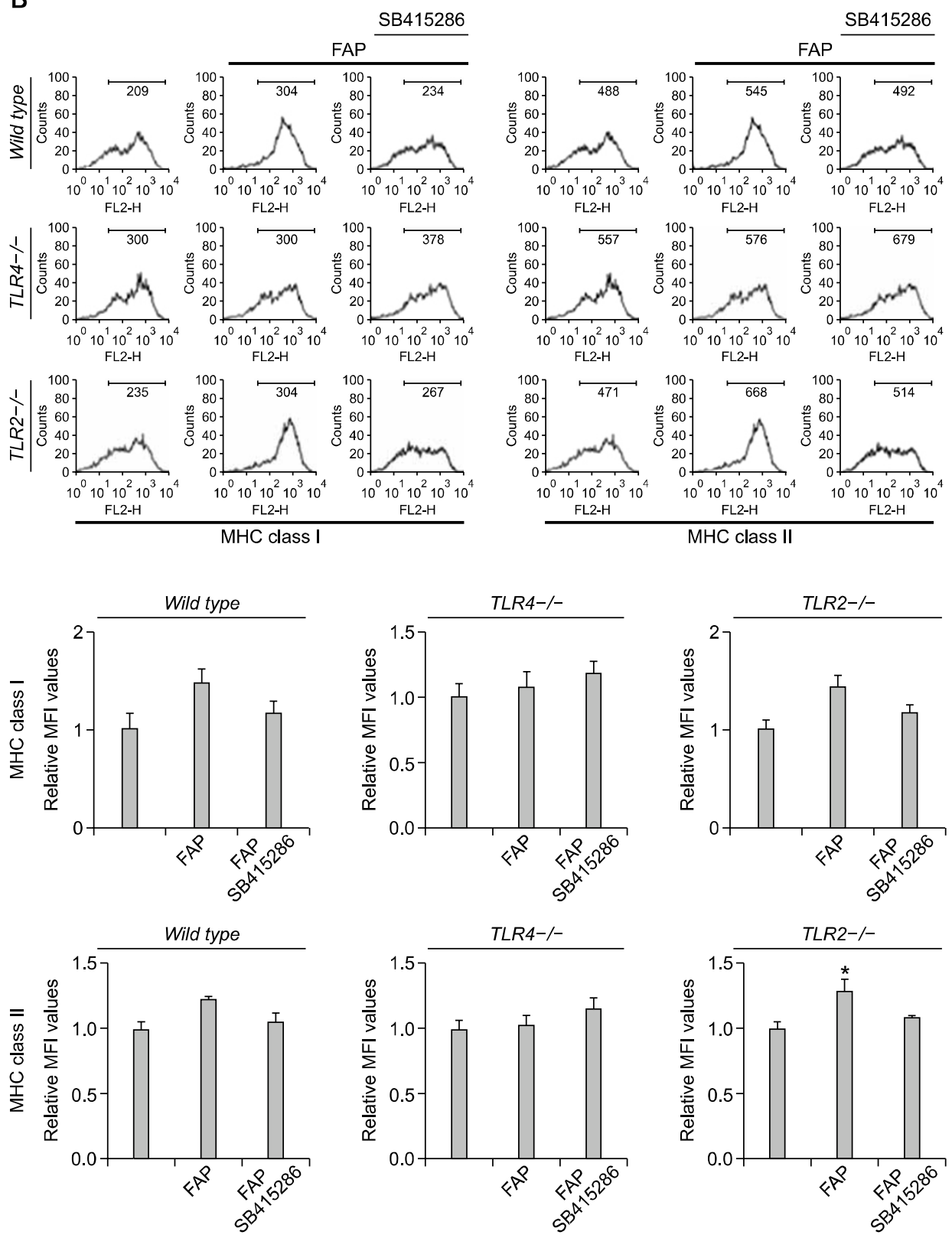

Figure 3. Continued.

FAP-treated DCs induce proliferation and CTL activity of CD $8^{+}$T-cell via GSK-3 activity

To determine whether the FAP-induced maturation of DCs affects T-cell activation, we performed a mixed lymphocyte reaction (MLR) assay using $\mathrm{CD}^{+}{ }^{\mathrm{T}}$-cells of OT-1 TCR transgenic mice, which express a TCR specific for the MHC class I-restricted Ovalbumin (OVA) peptide 257-264 antigen $\left(\mathrm{OVA}_{257-264}\right)$ in DCs (Hogquist et al., 1994). The proliferation of CFSE- labeled OVA-specific $\mathrm{CD} 8^{+}$ T-cells co-cultured with FAP-treated DCs pulsed with $\mathrm{OVA}_{257-264}$ was significantly higher than that of T-cells co-cultured with untreated DCs pulsed with
$\mathrm{OVA}_{257-264}$ (Figure 4A). In addition, the FAP-mediated enhanced $\mathrm{CD} 8^{+}$T-cell proliferation was dramatically inhibited by the treatment with the GSK-3 inhibitor SB415286 (Figure 4A). Above FAP-mediated $\mathrm{CD}^{+}{ }^{+}$T-cell proliferation was not observed in the absence of TLR4 (Supplemental Data Figure S2). These results show that FAP functions as an immunostimulator for DC-mediated $\mathrm{CD}^{+}$T-cell proliferation via TLR4- GSK-3 signaling cascade.

Next, we investigated whether FAP-mediated $\mathrm{CD} 8^{+}$T-cell proliferation causes cytotoxic $\mathrm{T}$ lymphocyte (CTL) responses. OVA-pulsed DCs 
A
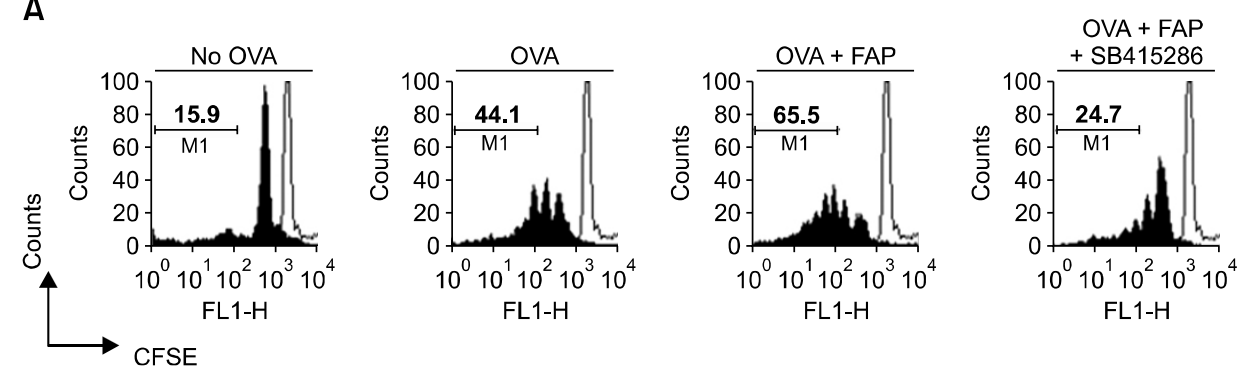

B
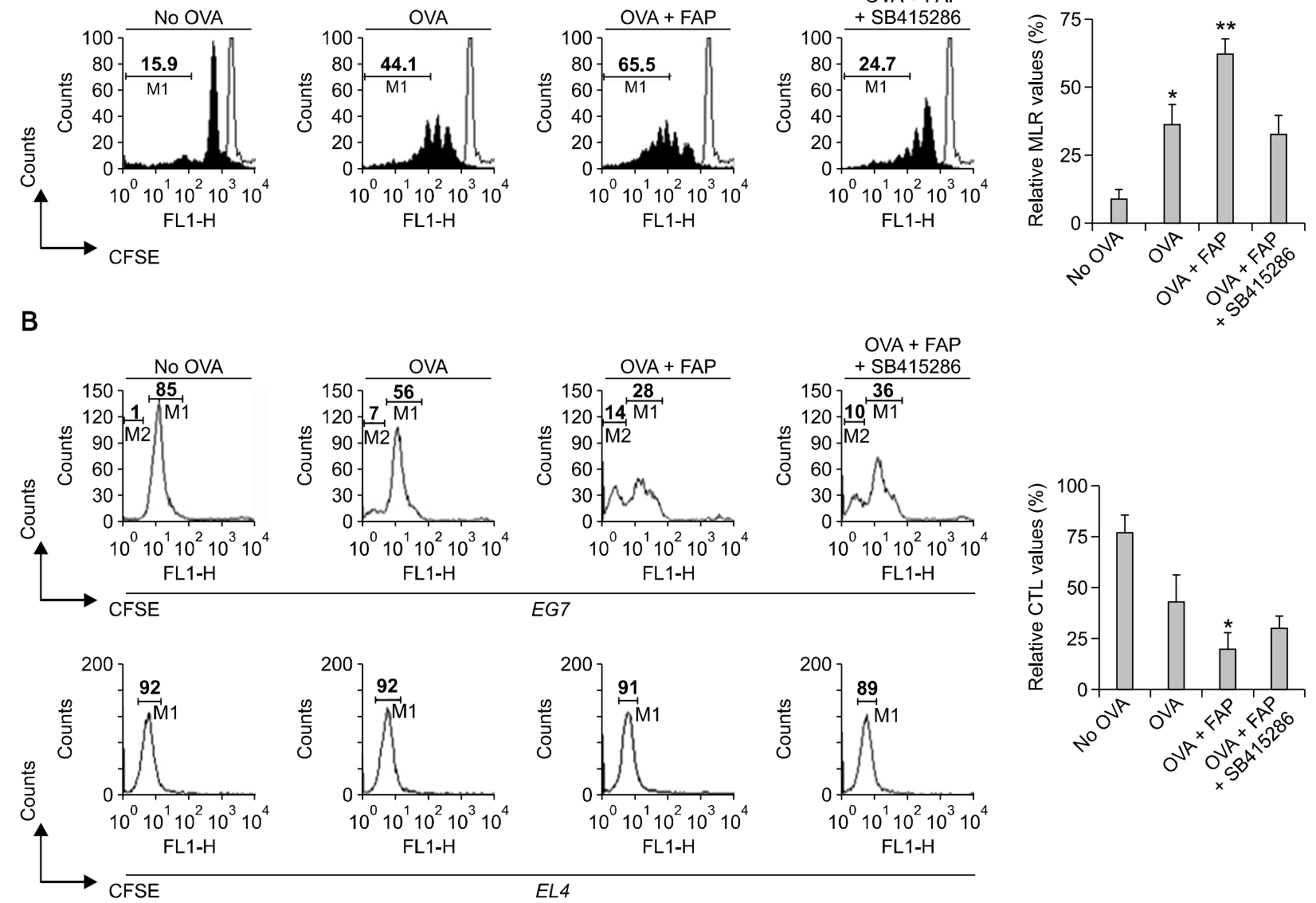

Figure 4. FAP-treated DCs induce proliferation and CTL activity of CD $8^{+}$T-cells via GSK-3 activity. (A) Immature DCs, OVA-pulsed DCs, OVA-pulsed FAP-treated DCs, or OVA-pulsed FAP + GSK-3 inhibitor-treated DCs were cultured with CFSE-labeled splenocytes of OT-1 T-cell receptor transgenic mice $\left(1 \times 10^{6}\right.$ per well) for $72 \mathrm{~h}$. After 3 days, the cells were harvested and stained with Cy5-labeled anti-CD8 monoclonal Ab and analyzed by flow cytometry. Histograms showing $\mathrm{CD}^{+}$T-cell proliferation as assessed by flow cytometry. The mean \pm SEM values shown represent 3 independent experiments. ${ }^{*} P<0.05$, ${ }^{* *} P<0.01$ compared with immature DCs. (B) In mixed cultures, immature DCs, OVA-pulsed DCs, OVA-pulsed FAP-treated DCs, or OVA-pulsed FAP + GSK-3 inhibitor-treated DCs were cultured with splenocytes of OT-1 T-cell receptor transgenic mice $\left(1 \times 10^{6}\right.$ per well) for 72 $\mathrm{h}$ and then co-cultured with EL4 $\left(1 \times 10^{6}\right.$ cells stained with $10 \mu \mathrm{M}$ CFSE) or EG7 $\left(1 \times 10^{6}\right.$ cells stained with $\left.10 \mu \mathrm{M} \mathrm{CFSE}\right)$ cells. After 4 hours, mixed lymphocyte tumor cultures were analyzed by flow cytometry. The percentage in M1 indicates CFSE-stained EG7 cells and that in M2 indicates lysed cell debris of CFSE-stained EG7 cells. The mean \pm SEM values shown represent 3 independent experiments. ${ }^{*} P<0.05$ compared with immature DCs.

reduced the population of CSFE-stained OVAexpressing EG7 tumor cells compared to non-pulsed DCs, and OVA-expressing EG7 tumor cells were significantly lysed in FAP-treated DCs pulsed with $\mathrm{OVA}_{257-264}$ (Figure 4B, upper). Furthermore, this increased CTL activity by FAP was inhibited following GSK-3 inhibition (Figure 4B, upper). However, FAP-mediated modulation of DCs did not affect the CTL responses against non-OVA-expressing EL4 tumor cells (Figure 4B, bottom). From these data, we concluded that FAP-treated DCs pulsed with $\mathrm{OVA}_{257-264}$ potentiates the OVA-specific CTL activity via the GSK-3 signaling pathway.
FAP triggers DC-derived anti-tumor activity in vivo Among the many TLR agonists, TLR4 agonists have a promising potential as immunostimulatory adjuvants because TLR4 signaling strongly induces DC maturation (Kaisho et al., 2001). In addition, it has been well documented that the expression of TLR4 on DCs is crucial for the anti-tumor effects of DC-based immunotherapy (Cisco et al., 2004). To determine the anti-tumor potential of immunization with FAP-treated DCs, mice were intraperitoneally injected 3 times (1 week apart) with immature DCs and OVA (1 $\mu \mathrm{g} / \mathrm{ml})$-pulsed DCs $\left(1 \times 10^{6}\right)$ treated with or without FAP $(500 \mathrm{ng} / \mathrm{ml})$, followed by subcutaneous injection of EG7 thymoma cells $\left(1 \times 10^{6}\right)$ into the right lower back. Injection of DCs pulsed with 
A

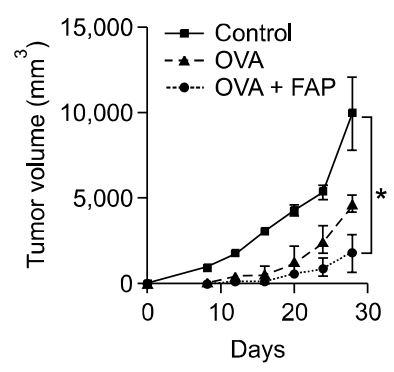

B

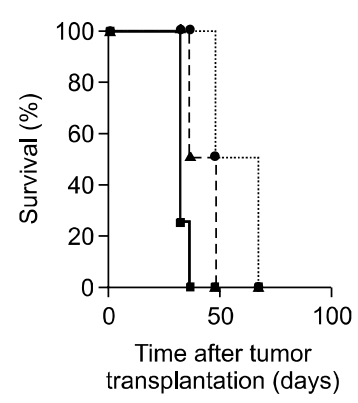

Figure 5. FAP triggers DC-derived anti-tumor activity in vivo. (A) Mice were intraperitoneally injected 3 times (1 week apart) with immature DCs and OVA $(1 \mu \mathrm{g} / \mathrm{ml})$-pulsed DCs $\left(1 \times 10^{6}\right)$ treated with or without FAP, followed by subcutaneous injection of EG7 thymoma cells $\left(1 \times 10^{6}\right)$ into the right lower back. The tumor size was measured every 4 days, and the tumor mass was calculated. $n=4$ mice/group. ${ }^{*} P<0.05$. (B) Survival of mice was recorded for up to 100 days, $n=4$ mice/group.

$\mathrm{OVA}_{257-264}$ significantly reduced the size of the OVA-expressing EG7 tumors compared to tumors in mice receiving $D C s$ that have not been pulsed with OVA (Figure 5A). Interestingly, EG7 tumors in mice that received FAP-treated DCs pulsed with OVA were smaller than those in mice that received DCs pulsed with OVA (Figure 5A). Consistent with this, the group of mice injected with FAP-treated OVA-pulsed DCs showed a significant prolongation of survival compared to mice injected with OVA-pulsed DCs or DCs that have not been pulsed with OVA (Figure 5B).

\section{Discussion}

Recently, studies on the regulation of the immune response by $M$. avium subsp. paratuberculosis have been reported (Lei et al., 2007; Lee et al., 2009). It is well known that $M$. avium subsp. paratuberculosis is crucial for Johne's disease in animals. In addition, on the basis of recent clinical trials, it is considered a possible cause of Crohn's disease, an inflammatory disease of the intestines. Crohn's disease is considered an autoimmune disease because the immune system attacks the gastrointestinal tract, causing chronic inflammation (Abraham and Cho, 2009).

DCs function as a crucial player in the innate and adaptive immune response. Thus, anti-tumor immune responses to DC-based vaccination have been suggested and studied with regard to the above-mentioned DC functions. In this study, we demonstrated following novel properties of the $M$. avium subsp. paratuberculosis FAP. 1) FAP-mediated DC maturation is via TLR4. 2) FAP-mediated DC

maturation is via GSK-3. 3) FAP could be utilized for DC- based cancer vaccine as an immuneenhancement adjuvant.

TLR4 agonists have a broad spectrum of immunoregulatory applications, e.g., as adjuvants for vaccine treatment of chronic viral infections and in tumor therapy (Cisco et al., 2004). Although LPS, a representative agonist of TLR4, is appropriate for immune enhancement, it is not applicable for anti-tumor immunity due to the risk of endotoxemia. In a previous study, we had shown that FAP is less toxic to DCs in vitro (Lee et al., 2009). Due to the non-toxic effects on DCs, we suggest that FAP is a potentially suitable adjuvant for immunotherapy.

Although $M$. avium subsp. paratuberculosis is a dangerous pathogen threatening animals and humans, FAP is applicable for the enhancement of immunogenicity because of its immunostimulatory ability. Our study provides for the first time evidence that FAP is an efficient TLR4 agonist. Further research to elucidate the exact mechanism underlying the regulation of DCs by FAP is currently underway. A more precise understanding of the interrelationship between DCs and M. avium subsp. paratuberculosis will provide crucial information for the therapy of cancer.

\section{Methods}

\section{Animals and reagents}

We purchased 4- to 8-week-old male C57BL/6 mice from the Korean Institute of Chemistry Technology (Daejeon, Korea). C57BL/6 OT-1 T-cell receptor (TCR) transgenic

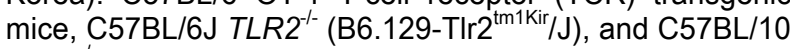
$T L R 4^{-1-}(\mathrm{C} 57 \mathrm{BL} / 10 \mathrm{ScNJ})$ were purchased at 6-8 weeks of age from the Jackson Laboratory (Bar Harbor, ME). All mice were housed in a specific pathogen-free environment and used in accordance with the institutional guidelines for animal care. Recombinant murine granulocyte-macrophage colony-stimulating factor (rmGM-CSF) and rmlL-4 were purchased from R\&D Systems (Minneapolis, MN). Cytokine enzyme-linked immunosorbent assay (ELISA) kits for murine IL-12p70, tumor necrosis factor-alpha $(T N F-\alpha)$, and IL-10 were purchased from R\&D Systems. Fluorescein isothiocyanate (FITC)- or phycoerythrin (PE)-conjugated monoclonal antibodies (mAbs) used to detect the expression of TLR4, CD11c (HL3), CD80 (16-10A1), CD86 (GL1), I-A $\mathrm{A}^{\mathrm{b}} \beta$-chain (AF-120.1), and H-2K ${ }^{\mathrm{b}}$ (AF6-88.5) by flow cytometry, as well as isotype-matched control mAbs and the biotinylated anti-CD11c (N418) mAb were purchased from R\&D Systems. Alexa568-conjugated rabbit IgG $A b$ and anti-mouse TLR4/MD-2 complex Ab were purchased from eBioscience, Inc. (San Diego, CA). Anti-phospho-ERK, anti-ERK, anti-phospho-p38, anti-phospho-JNK, and anti-I-кB Abs were purchased from Santa Cruz Biotechnology (Santa Cruz, CA). 


\section{Cell lines}

The cell lines EL4, a thymoma-derived cell line, and EG7, an OVA-expressing EL4 variant were purchased from the American Type Culture Collection (ATCC; Manassas, VA) and cultured in RPMI 1640 supplemented with 10\% heat-inactivated fetal bovine serum (FBS), $100 \mathrm{U} / \mathrm{ml}$ penicillin, $100 \mu \mathrm{g} / \mathrm{ml}$ streptomycin, and $10 \mathrm{mM}$ L-glutamine (all purchased from Invitrogen, Carlsbad, CA) at $37^{\circ} \mathrm{C}$ in a $5 \%$ $\mathrm{CO}_{2}$ atmosphere.

\section{Expression and purification of recombinant FAP}

Preparation of recombinant FAP has been described previously (Cho et al., 2007). Briefly, genomic DNA was isolated from the $M$. avium subsp. paratuberculosis JTC303 strain, and the DNA encoding FAP (MAP1569) was amplified by PCR. After purification of the PCR product, it was ligated into the $\mathrm{pET}-22 \mathrm{~b}(+)$ vector digested with the Ndel and Xhol enzymes. Expression of FAP in transformed BL21(DE3) cells was induced with isopropylbeta-D-thiogalactopyranoside (IPTG) (Promega, Madison, $\mathrm{WI})$. The soluble FAP was extracted after cell disruption by sonication. FAP containing a C-terminal histidine tag was purified using Ni-nitrilotriacetic acid (NTA) resin (Qiagen, Chatsworth, CA). The FAP was dialyzed five times in 10 $\mathrm{mM}$ phosphate-buffered saline (PBS) $(\mathrm{pH} 7.2)$ using a Slide-A-Lyzer dialysis cassette with a 3-kDa cutoff (Pierce, Rockford, IL). After dialysis, contaminating endotoxin was removed using Detoxi-Gel Affinity Pak columns (Pierce, Rockford, IL). FAP was incubated with endotoxin removal resin overnight to remove LPS and concentrated with a Centricon device (2,000-Da cutoff; Millipore). Also, endotoxin was assayed under endotoxin-free experimental conditions using a Limulus amebocyte lysate pyrogen kit (Biowhittaker, Walkersville, MD). The experiments were conducted according to the manufacturer's protocol. The quantity of endotoxin in the FAP was $\leq 0.01 \mathrm{ng} / \mathrm{mg}$. The final concentration of purified FAP was determined using a bicinchoninic acid protein assay kit (Pierce, Rockford, IL).

\section{Generation and culture of DCs}

Primary culture of DCs was performed as previously described (Jung et al., 2010), with slight modification. Briefly, bone marrow (BM) was flushed from the tibiae and femurs of 6 to 8-week-old male C57BL/6 mice and depleted of red blood cells (RBCs) using a red blood cell lysing buffer (Sigma-Aldrich, St. Louis, MO). The cells were then plated in 6-well culture plates $\left(1 \times 10^{6}\right.$ cells $/ \mathrm{ml} ; 2 \mathrm{ml}$ per well) in RPMI 1640 supplemented with $10 \%$ heat-inactivated FBS, $100 \mathrm{U} / \mathrm{ml}$ penicillin, $100 \mathrm{mg} / \mathrm{ml}$ streptomycin, $20 \mathrm{ng} / \mathrm{ml}$ $\mathrm{rmGM}-\mathrm{CSF}$, and $10 \mathrm{ng} / \mathrm{ml} \mathrm{rmlL}-4$ at $37^{\circ} \mathrm{C}$ in $5 \% \mathrm{CO}_{2}$. On days 3 and 5 , the floating cells were gently removed from the cultures and fresh medium was added. On day 6 of the culture, non-adherent cells and loosely adherent proliferating DC aggregates were harvested and re-plated in $60-\mathrm{mm}$ dishes $\left(1 \times 10^{6}\right.$ cells $\left./ \mathrm{ml} ; 5 \mathrm{ml} / \mathrm{dish}\right)$ for stimulation and analysis. On day $7,80 \%$ or more of the non-adherent cells expressed CD11c. In certain experiments, the DCs were labeled with bead-conjugated anti-CD11c mAb (Miltenyi Biotec, Gladbach, Germany) and then subjected to positive selection through paramagnetic columns (LS columns;
Miltenyi Biotec), according to the manufacturer's instructions, to obtain highly-purified populations for subsequent analysis. The purity of the selected cell fraction was $>90 \%$.

\section{Flow cytometric analysis}

On day 7, the BMDCs were harvested, washed with phosphate buffered saline (PBS), and resuspended in FACS washing buffer ( $2 \%$ FBS and $0.1 \%$ sodium azide in PBS). The cells were first blocked with $10 \%(\mathrm{v} / \mathrm{v})$ normal goat serum for 15 min at $4^{\circ} \mathrm{C}$ and then stained with $\mathrm{PE}$-conjugated anti-H-2K ${ }^{\mathrm{b}}$, anti-I-A $\mathrm{A}^{\mathrm{b}}$, anti-CD80, anti-CD86, anti-MHC class $\mathrm{I}$, anti-MHC class II, or with FITC-conjugated anti-CD11C for $30 \mathrm{~min}$ at $4^{\circ} \mathrm{C}$. Finally, the stained cells were analyzed using the FACSCalibur flow cytometer (Becton Dickinson, San Jose, CA).

\section{Mixed leukocyte reaction}

Transgenic OVA-specific $\mathrm{CD} 8^{+}$T-cells were purified from bulk splenocytes via negative selection by using a mouse $\mathrm{CD}^{+}$T-cell kit (Miltenyi Biotec). The purity of the obtained cell population was assessed to be $>93 \%$ by flow cytometry after staining with a Cy5-conjugated anti-CD8 Ab. Briefly, the cells were resuspended in $5 \mu \mathrm{M}$ carboxyfluorscein diacetate succinimidyl ester (CFSE) in DMSO and shaken for $10 \mathrm{~min}$ at room temperature. Next, the cells were washed once in pure FBS and twice in PBS with $10 \%$ FBS. DCs $\left(1 \times 10^{5}\right)$ were then exposed to FAP for $24 \mathrm{~h}$ and subsequently co-cultured with $1 \times 10^{6} \mathrm{CFSE}$-labeled T lymphocytes in 96-well, U-bottom plates. After 3 days, the cells were harvested, stained with a Cy5-labeled anti-CD8 monoclonal $\mathrm{Ab}$ (to gate OT-1 T-cells), and then assessed by flow cytometry.

\section{In vitro cytotoxicity assays}

To determine the effect of FAP on the induction of OVA-specific CTLs, cytotoxicity assays were performed. In mixed cultures, immature DCs, OVA-pulsed DCs, or OVA-pulsed FAP-treated DCs were first cultured with splenocytes of OT- 1 TCR transgenic mice $\left(1 \times 10^{6}\right.$ per well $)$ for $72 \mathrm{~h}$ and then co-cultured with EL4 $\left(1 \times 10^{6}\right.$, cells stained with $1 \mu \mathrm{M}$ CFSE) or EG7 cells $\left(1 \times 10^{6}\right.$ cells stained with $10 \mu \mathrm{M}$ CFSE). After $4 \mathrm{~h}$, the mixed lymphocyte tumor cultures were analyzed by flow cytometry.

\section{Cytokine assay}

Murine IL-12p70, TNF- $\alpha$, and IL-10 were measured using an ELISA kit according to the manufacturer's instructions.

\section{Confocal laser scanning microscopy}

The cells were permeabilized with $1 \%$ saponin for $3 \mathrm{~min}$, stained with rabbit anti-FAP and rat anti-TLR4/MD2 Abs overnight at $4^{\circ} \mathrm{C}$, and then stained with rabbit anti-FAP and mouse PE-conjugated anti-TLR4 antibodies overnight at $4^{\circ} \mathrm{C}$, and then stained with Alexa568-conjugated anti-rabbit antibody for $1 \mathrm{~h}$ at room temperature. Cell morphology and 
fluorescence intensity were analyzed using the Zeiss LSM510 Meta confocal laser scanning microscope (Zeiss, Jena, Germany). Images were acquired using the LSM510 Meta software and processed using the LSM image examiner.

\section{Co-immunoprecipitation analysis}

Co-immunoprecipitation analysis was performed as previously described (Noh et al., 2010), with slight modification. The cells were lysed in lysis buffer $(0.5 \%$ NP-40, 1 mM EDTA, 50 mM Tris- $\mathrm{HCl}$, pH 8.0, $120 \mathrm{mM}$ $\mathrm{NaCl}$ ), and the lysates were subjected to $10 \mathrm{~min}$ of microcentrifugation at $12,000 \mathrm{~g}$. The supernatant was then incubated for $2 \mathrm{~h}$ at $4^{\circ} \mathrm{C}$ with the anti-FAP Ab, after which the mixture was incubated for an additional $1 \mathrm{~h}$ at $4^{\circ} \mathrm{C}$ with protein G-coupled sepharose beads (Amersham Pharmacia Biotech, Uppsala, Sweden). The resultant precipitates were subjected to SDS-PAGE and immunoblotted with anti-TLR4 Ab.

\section{Immunoblot analysis}

In brief, cell lysates were subjected to SDS-PAGE and transferred to PVDF membranes. The PVDF membranes were then blocked with $5 \%$ nonfat milk in a washing buffer (50 mM Tris- $\mathrm{HCl}, \mathrm{pH} 8.0,150 \mathrm{mM} \mathrm{NaCl}, 0.1 \%$ Tween 20) and incubated with the indicated antibodies for $1 \mathrm{~h}$ at room temperature. The membranes were washed and incubated for $1 \mathrm{~h}$ at room temperature with the appropriate secondary antibodies conjugated with horseradish peroxidase (Amersham Pharmacia Biotech, Uppsala). Protein bands were visualized using an enhanced chemiluminescence system (Amersham Pharmacia Biotech, Uppsala).

\section{Statistical analysis}

All experiments were repeated at least 3 times with consistent results. Unless otherwise stated, data are expressed as the mean \pm SEM. Analysis of variance was used to compare experimental groups with control values, while comparisons between multiple groups were made using Tukey's multiple comparison tests (Prism 3.0 GraphPad software). A $P$ value less than 0.05 was considered to indicate statistical significance.

\section{Supplemental data}

Supplemental Data include two figures and can be found with this article online at http://e-emm.or.kr/article/article_ files/ SP-44-5-04.pdf.

\section{Acknowledgements}

This study was supported by a grant of the Korea Healthcare Technology R\&D Project, Ministry for Health Welfare \& Family Affairs, Republic of Korea (A091047).

\section{References}

Abraham C, Cho JH. Inflammatory bowel disease. N Engl J Med 2009;361:2066-78

Akira S, Takeda K, Kaisho T. Toll-like receptors: critical proteins linking innate and acquired immunity. Nat Immunol 2001;2:675-80

Alvarez D, Vollmann EH, von Andrian UH. Mechanisms and consequences of dendritic cell migration. Immunity 2008;29:325-42

Banchereau J, Briere F, Caux C, Davoust J, Lebecque S, Liu YJ, Pulendran B, Palucka K. Immunobiology of dendritic cells. Annu Rev Immunol 2000;18:767-811

Cho D, Shin SJ, Talaat AM, Collins MT. Cloning, expression, purification and serodiagnostic evaluation of fourteen Mycobacterium paratuberculosis proteins. Protein Expr Purif 2007;53;411-20

Cisco RM, Abdel-Wahab Z, Dannull J, Nair S, Tyler DS, Gilboa E, Vieweg J, Daaka, Y, Pruitt SK. Induction of human dendritic cell maturation using transfection with RNA encoding a dominant positive toll-like receptor 4 . J Immunol 2004;172:7162-8

Cohen P, Frame S. The renaissance of GSK3. Nat Rev Mol Cell Biol 2001;2:769-76

Hogquist KA, Jameson SC, Heath WR, Howard JL, Bevan MJ, Carbone FR. T cell receptor antagonist peptides induce positive selection. Cell 1994;76:17-27

Jensen SS, Gad M. Differential induction of inflammatory cytokines by dendritic cells treated with novel TLR-agonist and cytokine based cocktails: targeting dendritic cells in autoimmunity. J Inflamm (Lond) 2010;7:37

Jope RS, Johnson GV. The glamour and gloom of glycogen synthase kinase-3. Trends Biochem Sci 2004;29:95-102

Jung ID, Noh KT, Lee CM, Chun SH, Jeong SK, Park JW, Park WS, Kim HW, Yun C H, Shin YK, Park YM. Oncostatin M induces dendritic cell maturation and Th1 polarization. Biochem Biophys Res Commun 2010;394:272-8

Kaisho T, Akira S. Dendritic-cell function in Toll-like receptorand MyD88-knockout mice. Trends Immunol 2001;22:78-83

Kuroda K, Brown EJ, Telle WB, Russell DG, Ratliff TL. Characterization of the internalization of bacillus Calmette-Guerin by human bladder tumor cells. J Clin Invest 1993:91:69-76

Lee JS, Shin, SJ, Collins MT, Jung ID, Jeong YI, Lee CM, Shin YK, Kim D, Park YM. Mycobacterium avium subsp. paratuberculosis fibronectin attachment protein activates dendritic cells and induces a Th1 polarization. Infect Immun 2009;77:2979-88

Lei L, Hostetter JM. Limited phenotypic and functional maturation of bovine monocyte-derived dendritic cells following Mycobacterium avium subspecies paratuberculosis infection in vitro. Vet Immunol Immunopathol 2007;120: 177-86

Liu YJ. Dendritic cell subsets and lineages, and their functions in innate and adaptive immunity. Cell 2001;106: 


\section{9-62}

Martin M, Rehani K, Jope RS, Michalek SM. Toll-like receptor-mediated cytokine production is differentially regulated by glycogen synthase kinase 3 . Nat Immunol 2005;6:777-84

Matijevic T, Pavelic J. Toll-like receptors: cost or benefit for cancer? Curr Pharm Des 2010;16:1081-90

Middleton AM, Chadwick MV, Nicholson AG, Dewar A Groger RK, Brown EJ, Wilson R. The role of Mycobacterium avium complex fibronectin attachment protein in adherence to the human respiratory mucosa. Mol Microbiol 2000;38: $381-91$

Napolitani G, Rinaldi A, Bertoni F, Sallusto F, Lanzavecchia A. Selected Toll-like receptor agonist combinations synergistically trigger a $T$ helper type 1-polarizing program in dendritic cells. Nat Immunol 2005;6:769-76

Noh KT, Cho SG, Choi EJ. Knockdown of apoptosis signal-regulating kinase 1 modulates basal glycogen synthase kinase- $3 \beta$ kinase activity and regulates cell migration. FEBS Lett 2010;584:4097-101

Noh KT, Park YM, Cho SG, Choi EJ. GSK-3beta-induced ASK1 stabilization is crucial in LPS-induced endotoxin shock. Exp Cell Res 2011;317:1663-8

Ratliff TL, McCarthy R, Telle WB, Brown EJ. Purification of a mycobacterial adhesin for fibronectin. Infect Immun 1993; 61:1889-94

Rescigno M, Martino M, Sutherland CL, Gold MR, Ricciardi-Castagnoli P. Dendritic cell survival and maturation are regulated by different signaling pathways. J Exp Med 1998;188:2175-80

Rodionova E, Conzelmann M, Maraskovsky E, Hess M, Kirsch M, Giese T, Ho AD, Zoller M, Dreger P, Luft T. GSK-3 mediates differentiation and activation of proinflammatory dendritic cells. Blood 2007;109:1584-92

Schorey JS, Holsti MA, Ratliff TL, Allen PM, Brown EJ. Characterization of the fibronectin-attachment protein of Mycobacterium avium reveals a fibronectin-binding motif conserved among mycobacteria. Mol Microbiol 1996;21: $321-9$

Schorey JS, Li Q, McCourt DW, Bong-Mastek M, Clark-Curtiss JE, Ratliff TL, Brown EJ. A Mycobacterium leprae gene encoding a fibronectin binding protein is used for efficient invasion of epithelial cells and Schwann cells. Infect Immun 1995;63:2652-7

Secott TE, Lin TL, Wu CC. Fibronectin attachment protein homologue mediates fibronectin binding by Mycobacterium avium subsp. paratuberculosis. Infect Immun 2001;69: 2075-82

Watts C, West MA, Zaru R. TLR signalling regulated antigen presentation in dendritic cells. Curr Opin Immunol 2010;22:124-30

Woodgett JR. Judging a protein by more than its name: GSK-3. Sci STKE 2001 2001;re12

Zhao W, Schorey JS, Bong-Mastek M, Ritchey J, Brown EJ, Ratliff TL. Role of a bacillus Calmette-Guerin fibronectin attachment protein in BCG-induced antitumor activity. Int $\mathrm{J}$ Cancer 2000;86:83-8 